\title{
De-regulated STAT5A/miR-202-5p/USP15/ Caspase-6 regulatory axis suppresses CML cell apoptosis and contributes to Imatinib resistance
}

Zi-Yuan Nie ${ }^{1}$, Min Yao ${ }^{2}$, Zhan Yang ${ }^{2,3}$, Lin Yang ${ }^{1}$, Xiao-Jun Liư ${ }^{1}$ Jing Yu², Ying Ma², Nan Zhang ${ }^{2}$, Xiao-Yan Zhang ${ }^{1}$, Meng-Han Liu', Ling-Ling Jiang ${ }^{2^{*}}$ and Jian-Min Luo ${ }^{1^{*}}$

\begin{abstract}
Background: STAT5 plays an important role in the transformation of hematopoietic cells by BCR-ABL. However, the downstream target genes activated by STAT5 in chronic myeloid leukemia (CML) cells remain largely unclear. Here, we investigated the mechanistic functional relationship between STAT5A-regulated microRNA and CML cell apoptosis.

Methods: The expression of USP15, Caspase-6, STAT5A-regulated miR-202-5p and STAT5A was detected by qRTPCR and Western blotting in CML cell lines and PBMCs of CML patients. Cell apoptosis was evaluated by flow cytometry. Both gain- and loss-of-function experiments were used to investigate the roles of USP15, miR-202-5p and STAT5A in CML. Luciferase reporter assay detected the effect of miR-202-5p on USP15 expression. Xenograft animal model was used to test the effect of anti-miR-202-5p and pimozide on K562 cell xenograft growth.
\end{abstract}

Results: USP15 expression was significantly downregulated in CML cell lines and PBMCs of CML patients. Depletion of USP15 increased, whereas overexpression of USP15 reduced the resistance of CML cells to Imatinib. Further, decreased deubiquitinating activity of USP15 by USP15 downregulation led to reduced caspase- 6 level, thus attenuating CML cell apoptosis. Mechanistically, miR-202-5p was upregulated in K562G cells and negatively regulated USP15 expression by directly targeting USP15 3'-UTR. Correspondingly, upregulation of miR-202-5p enhanced the resistance of CML cells to Imatinib by inhibiting cell apoptosis. Importantly, STAT5A was upregulated in CML cells and directly activated miR-202-5p transcription by binding to the pre-miR-202 promoter. Pimozide induced CML cell apoptosis and significantly reduced K562 cell xenograft growth in vivo by blocking STAT5A/miR202-5p/USP15/Caspase-6 regulatory axis.

Conclusions: we provide the first evidence that de-regulated STAT5A/miR-202-5p/USP15/Caspase-6 regulatory axis suppresses the apoptosis of CML cells, targeting this pathway might be a promising therapeutic approach for the treatment of CML.

Keywords: CML, USP15, STAT5A, miRNA, Caspase-6

\footnotetext{
* Correspondence: jianglinglinghebmu@126.com; luojianmin1960@126.com

${ }^{2}$ Department of Biochemistry and Molecular Biology, The Key Laboratory of Neural and Vascular Biology, Ministry of Education of China, Hebei Medical University, No. 361 Zhongshan E Rd, Shijiazhuang 050017, China

${ }^{1}$ Department of Hematology, The Second Hospital of Hebei Medical

University, 215 Heping W Rd, Shijiazhuang 050000, China

Full list of author information is available at the end of the article
}

(c) The Author(s). 2020 Open Access This article is distributed under the terms of the Creative Commons Attribution 4.0 International License (http://creativecommons.org/licenses/by/4.0/), which permits unrestricted use, distribution, and reproduction in any medium, provided you give appropriate credit to the original author(s) and the source, provide a link to the Creative Commons license, and indicate if changes were made. The Creative Commons Public Domain Dedication waiver (http://creativecommons.org/publicdomain/zero/1.0/) applies to the data made available in this article, unless otherwise stated. 


\section{Background}

Chronic myeloid leukemia (CML) is a clonal disease of pluripotent hematopoietic cells characterized by the expression of the BCR/ABL1 fusion gene, which encodes a constitutively active tyrosine kinase $\mathrm{BCR}-\mathrm{ABL}[1,2]$. The elevated activity of BCR-ABL tyrosine kinase initiates CML and approximately $30 \%$ of acute lymphoblastic leukemia (ALL) by stimulating proliferation signals, such as Ras, phosphoinositide 3-kinase (PI3K)/protein kinase $\mathrm{B}$ (AKT) and signal transducer and activator of transcriptions (STATs) as well as by inhibiting apoptosis signals, such as Ras-dependent signaling pathway $[3,4]$.

The deubiquitinating enzyme ubiquitin-specific peptidase 15 (USP15) regulates diverse cellular processes in cancer [5]. USP15 has been shown to stabilize TGF- $\beta$ receptor I and promote oncogenesis through the activation of TGF- $\beta$ signaling in glioblastoma [6], and the USP15 gene is also amplified myeloma and ovarian cancers [7, 8]. Currently, the identified targets for USP15 include numerous cancer-associated proteins, such as proapoptotic caspase-3 [9], the transforming growth factor beta receptor [6], p53 [5], human homolog of mouse double minute 2 (MDM2) [10], and $\beta$-catenin [11]. However, the mechanistic and functional links among STAT5-regulated microRNA, USP15 and target protein of USP15 in CML cells remain poorly understood.

The phosphorylation of STAT5A is essential for the transformation of hematopoietic cells by BCR-ABL and plays an important role in resistance to apoptosis through enhancing the expression of anti-apoptotic factor Bcl-XL [4]. BCR-ABL has been shown to directly induce a tyrosine-phosphorylation and dimerization of STAT5 followed by nuclear translocation of the STAT5 dimers that then bind to consensus sequences and promote transcription of downstream target genes, such as TNFRSF13b, MKP-1, Bcl-XL, C3ar1, Cis, Spi2.1 and Socs-1 [12, 13]. Besides proliferation- and apoptosisrelated genes, STAT5 has also recently been implicated in the regulation of the expression of mammalian microRNA host genes. For example, STAT5 binds the promoter of the miR-22 host gene, and inhibition of JAK3, STAT3, and STAT5 increases expression of pri-miR-22 and miR-22, a novel tumor suppressor miRNA [14]. STAT5A/B enhances the cytokine-induced miR-193b transcription in hematopoietic stem cells (HSCs), thus providing a negative feedback for excessive signaling to restrict uncontrolled HSC expansion [15]. Despite these advances, it is largely unknown about how STAT5regulated microRNA affects CML development.

In the present study, we detected the expression of USP15, Caspase-6, STAT5A-regulated miR-202-5p and STAT5A in CML cell lines and PBMCs of CML patients and investigated the functional relationship between these gene expression and CML cell apoptosis in the context of Imatinib or pimozide treatment. Our findings provide the first evidence that de-regulated STAT5A/ miR-202-5p/USP15/Caspase-6 regulatory pathway suppresses the apoptosis of CML cells.

\section{Methods}

The detailed procedures of cell transfection, cell viability assay, analyses of apoptosis, xenograft animal model, RNA extraction and quantitative real-time PCR, western blot analysis, in situ hybridization, vector construction and luciferase reporter assay, immunofluorescence staining, Co-immunoprecipitation assay, Chromatin immunoprecipitation (ChIP) assay and highlight sequence of miRNA as well as key reagents are described in Additional file 2 .

\section{Patients and specimens}

30 patients with chronic phase of CML (CML-CP) who were admitted to the Department of Hematology of the Second Hospital of Hebei Medical University from May 2016 to June 2017 were selected as the research objects. 30 healthy donors were selected to serve as controls. Lymphocyte separation medium was used to isolate the peripheral blood mononuclear cells (PBMCs) according to the manufacturer's instructions. Diagnosis of $\mathrm{Ph}$ positive CML was confirmed by bone marrow morphology, cytogenetic and molecular biology. Before the specimens were collected, patients did not undergo any treatment. 6 PBMCs of patients with CML blast crisis (CML-BC) (4 patients with CML-AML and 2 patients with CML-ALL) was collected from the Department of Hematology of the Second Hospital of Hebei Medical University from May 2015 to December 2018. All the patients with CML-BC were absence with Imatinib until CML progresses and didn't have the BCR-ABL1 gene mutant. The characteristics of CML patients and healthy donors are summarized in Additional file 1: Table S2. The study protocol was approved by the Ethics Committee of Second Hospital of Hebei Medical University and written informed consent was obtained all the patients.

\section{Cell culture}

Human CML cell lines (KCL22 and K562) were maintained in the laboratory. The Imatinib-resistant $\mathrm{K} 562$ cells (K562G cell line) were established as previously described [16]. KCL22 cells were cultured in Iscove's modified Dulbecco's medium (IMDM; Gibco, Beijing, China), with 10\% fetal bovine serum (FBS) (Clark Bio, Claymont, DE, USA), $100 \mathrm{units} / \mathrm{ml}$ penicillin and $100 \mu \mathrm{g} / \mathrm{ml}$ streptomycin. K562, K562G cells were cultured in RPMI 1640 medium (Gibco, Beijing, China) with 10\% FBS and the two antibiotics listed above. Cell lines were grown at $37^{\circ} \mathrm{C}$ with $5 \% \mathrm{CO}_{2}$. The cell lines were characterized by Genetic Testing Biotechnology Corporation (Suzhou, 
China) using short tandem repeat (STR) markers. Short tandem repeat analysis was used to tested Mycoplasma contamination.

\section{Target prediction and bioinformatics analysis}

TargetScan (http://www.targetscan.org/vert_72/) were performed to identify the potential microRNAs target to 3'UTR of USP15. PROMO (http://alggen.lsi.upc.es) was used to search the potential transcriptional factor of premiR-202 and the potential element of STAT5A on the promoter region in pre-miR-202 promotor.

\section{Statistical analysis}

Data were presented as mean \pm SEM. Student's $t$ test was used to analyze differences between two groups. Spearman's correlation analysis was used to evaluate the correlation analysis. Values of $P<0.05$ were considered statistically significant. Graphpad Prism 7.0 software was using to perform the statistical analysis (GraphPad Software, San Diego, CA, USA).

\section{Results}

USP15 expression is significantly downregulated in CML

USP15 is previously reported to be dysregulated in many human cancers and plays critical roles in tumor development and progression [17]. Here, we first analyzed USP15 gene expression in different types of human leukemia using The Cancer Genome Atlas (TCGA) database. The results showed that the expression of USP15 was dramatically downregulated in acute leukemia including Acute Myeloid Leukemia (AML) and Acute Lymphoblastic Leukemia (ALL)comparing to the matched normal cells. A decreased USP15 expression was also found in CML but there was no significant difference between healthy donors and CML patients (Additional file 1: Fig. S1). Next, we examined USP15 mRNA

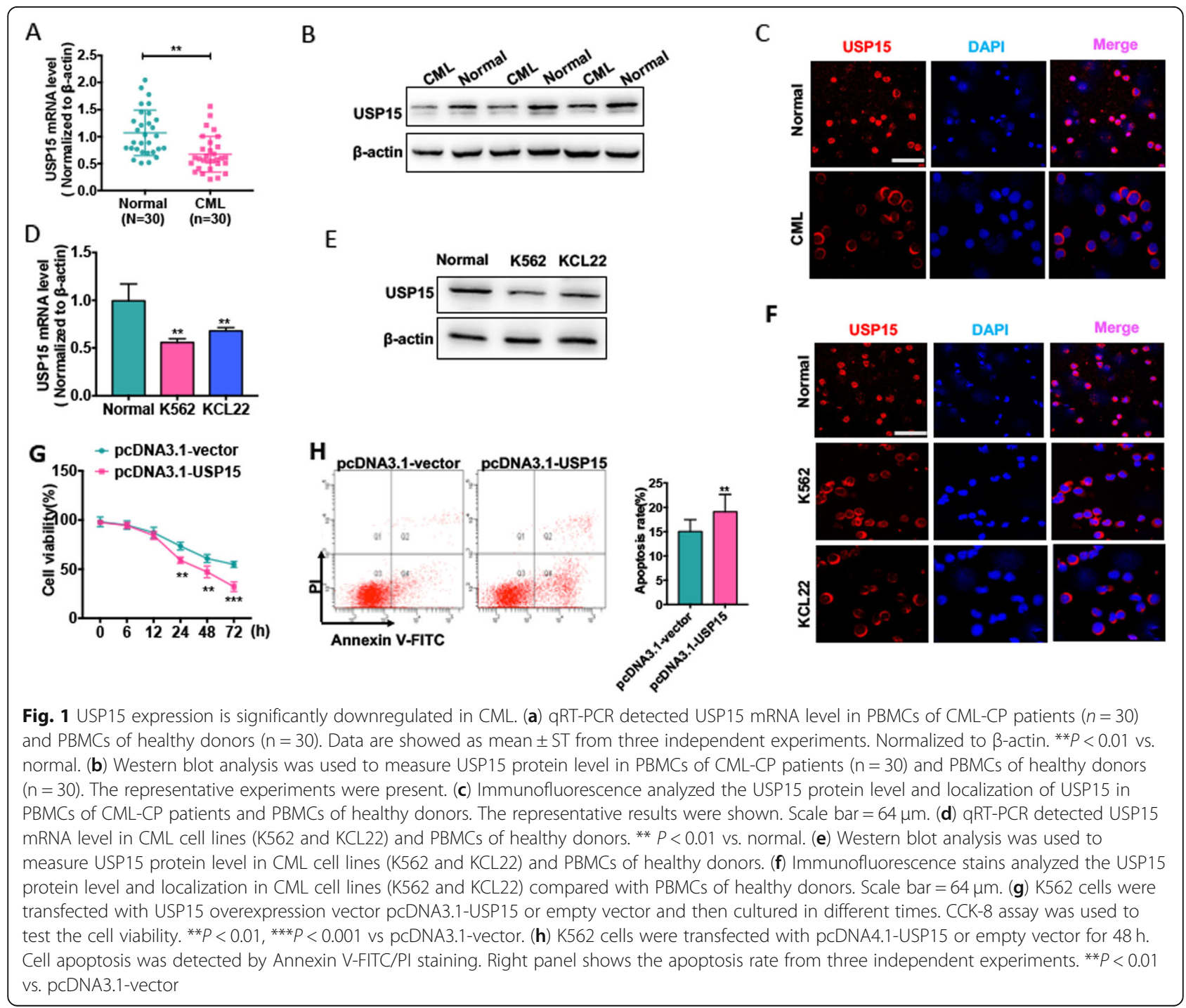


and protein expression levels in PBMCs of CML-CP patients and CML cell lines. We found that USP15 mRNA level was lower in PBMCs of CML patients than in healthy donors (Fig. 1 a). Importantly, the protein level of USP15 was significantly downregulated in PBMCs of CML patients compared with healthy donors (Fig. 1 b). Immunofluorescence staining revealed that USP15 is mainly localized in the nuclei of PBMCs in healthy donors, but it existed in the cytoplasm of PBMCs and its expression level was obviously reduced in PBMCs of CML patients (Fig. 1 c). Similarly, USP15 mRNA and protein levels were downregulated in CML cell lines (K562 and KCL22), as shown by Western blotting and qRT-PCR (Fig. $1 \mathrm{~d}$ and e). Immunofluorescence staining also confirmed that the changes of localization and expression of USP15 in CML cell lines were very similar to those seen in PBMCs of CML patients and healthy donors, consistent with those reported previously (Fig. $1 \mathrm{f}$ ) [18].

To further identify whether the downregulation of USP15 is correlated with CML cell proliferation and apoptosis, we overexpressed USP15 in K562 cells (Additional file 1: Fig. S2) and examined the effects of enforced USP15 expression on K562 cells. Expectedly, overexpression of USP15 significantly decreased the cell viability and thus increased the apoptosis of K562 cells (Fig. $1 \mathrm{~g}$ and $\mathrm{h}$ ). Together, these findings suggest that the downregulation of USP15 is responsible for increased CML cell proliferation and decreased apoptosis.

\section{Downregulation of USP15 contributes to Imatinib resistance of CML cells}

Because previously studies have reported that dysregulation of USP15 could result in paclitaxel resistance in HeLa cells [9], we wanted to investigate whether USP15 downregulation is associated with CML Imatinib resistance. As previously described, we first established an Imatinib-resistant K562 cell line (K562G cell line) and confirmed that the IC50 values of Imatinib in K562G cells were 20 folds higher than those in parental cell line (Additional file 1: Fig. S3). We next detected USP15 protein and mRNA levels in K562 and K562G cells. As shown in Fig. 2 a and b, the expression levels of USP15 mRNA and protein were much lower in K562G cells than in K562 cells. A similar result was obtained by immunofluorescence staining of USP15 (Fig. 2 c).

In addition, we analyzed the expression changes of USP15 in CML-BC patients who were confirmed to be secondary Imatinib resistance. The results showed that USP15 mRNA and protein levels were markedly decreased in PBMCs of CML-BC patients $(n=6)$ compared with age-matched CML-CP patients $(\mathrm{n}=6)$ (Fig. $2 \mathrm{~d}$ and e). These results indicate that Imatinib-resistant cell line and CML-BC patients have a decreased level of USP15 expression. Further, loss- and gain-of-function experiments were performed to investigate whether USP15 plays a role in Imatinib resistance of CML cells. As shown in Fig. $2 \mathrm{f}$, enforced USP15 expression in K562G cells significantly decreased the IC50 values of Imatinib and facilitated Imatinib-induced cell apoptosis, as evidenced by flow cytometry (Fig. 2 g). Conversely, USP15 downregulation in K562 cells dramatically increased the IC50 values of Imatinib and attenuated Imatinib-induced cell apoptosis (Fig. $2 \mathrm{~h}$ and i). These results indicate that depletion of USP15 increases, while overexpression of USP15 reduces the resistance of CML cells to Imatinib.

\section{Downregulation of USP15 decreases the apoptosis of CML cells by lowering the level of Caspase- 6 protein} USP15 is known to regulate the expression of multiple proteins at the post-translational level via ubiquitinproteasome pathway [19]. To clarify the downstream proteins of USP15 in CML cells, we performed coimmunoprecipitation coupled with mass spectrometry (CoIP-MS) and found that 25 proteins might be interacted with USP15 (Additional file 1: Table S1). Among these proteins, Caspase- 6 , as a major activator of Caspase-8 in vivo, has a critical role in promotion of apoptosis [20] and thus aroused our attention. we first analyzed the influence of USP15 on Caspase- 6 protein expression. As expected, USP15 overexpression markedly increased, whereas its knockdown reduced Caspase6 protein level in K562 cells (Fig. 3 a). Furthermore, a co-immunoprecipitation assay showed that there existed interaction between endogenous Caspase- 6 and USP15 (Fig. 3 b). Immunofluorescence staining also showed that endogenous USP15 and Caspase- 6 were co-localized in the cytoplasm of $\mathrm{K} 562$ cells (Fig. 3 c).

To further determine whether USP15 is involved in the regulation of Caspase- 6 degradation, we used proteasome inhibitor MG132 and lysosome inhibitor chloroquine (CQ) to treat K562 cells. The results showed that expression level of Caspase-6 protein was obviously increased in K562 cells treated with MG132, but not CQ, knockdown of USP15 in K562 cells partly decreased the upregulation of Caspase- 6 protein level by MG132 even in the presence to MG132, indicating that the ubiquitinproteasome pathway mediates the degradation of Caspase-6 (Fig. $3 \mathrm{~d}$ and Additional file 1: Fig. S4A). Additionally, K562 cells were transfected with si-USP15 or si$\mathrm{NC}$ and then treated with MG132 to suppress ubiquitinmediated degradation. Co-immunoprecipitation experiments results showed that knockdown of USP15 in K562 cells could significantly increase the of accumulation ubiquitinated Caspase-6 level compared with si-NC (Additional file 1: Fig. S4B), suggesting that USP15 positively regulates Caspase- 6 protein level of by promoting deubiquitination of Caspase- 6 and inhibiting the degradation 


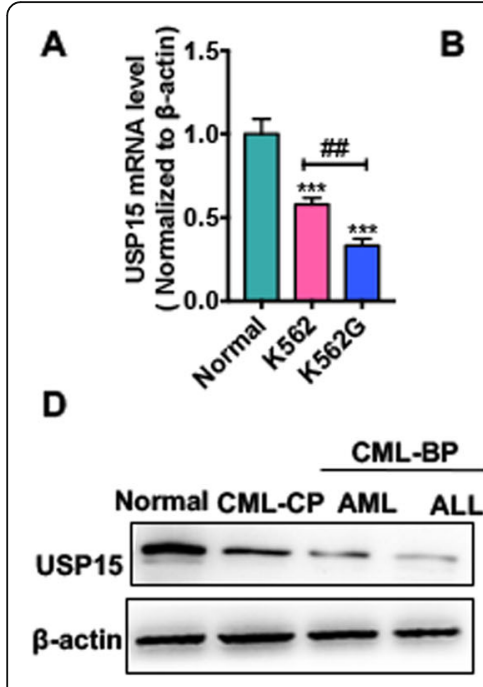

E

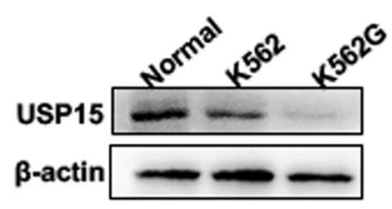

C
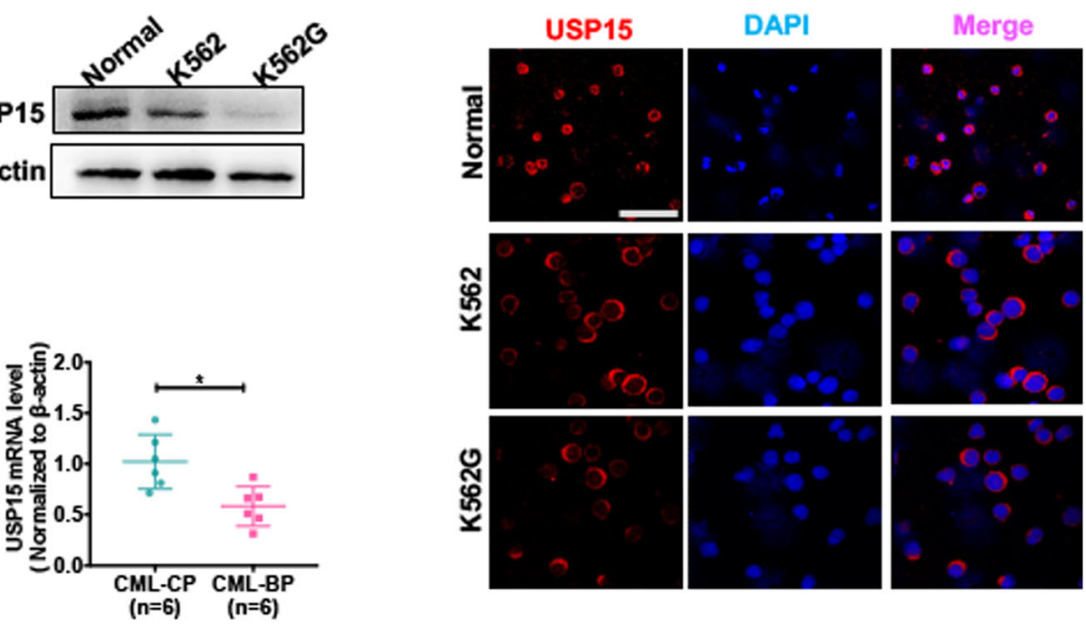

$\mathbf{F}$

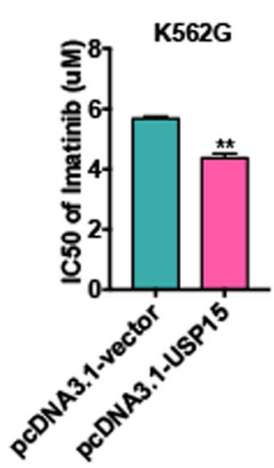

H

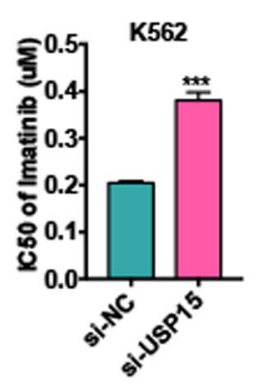

G
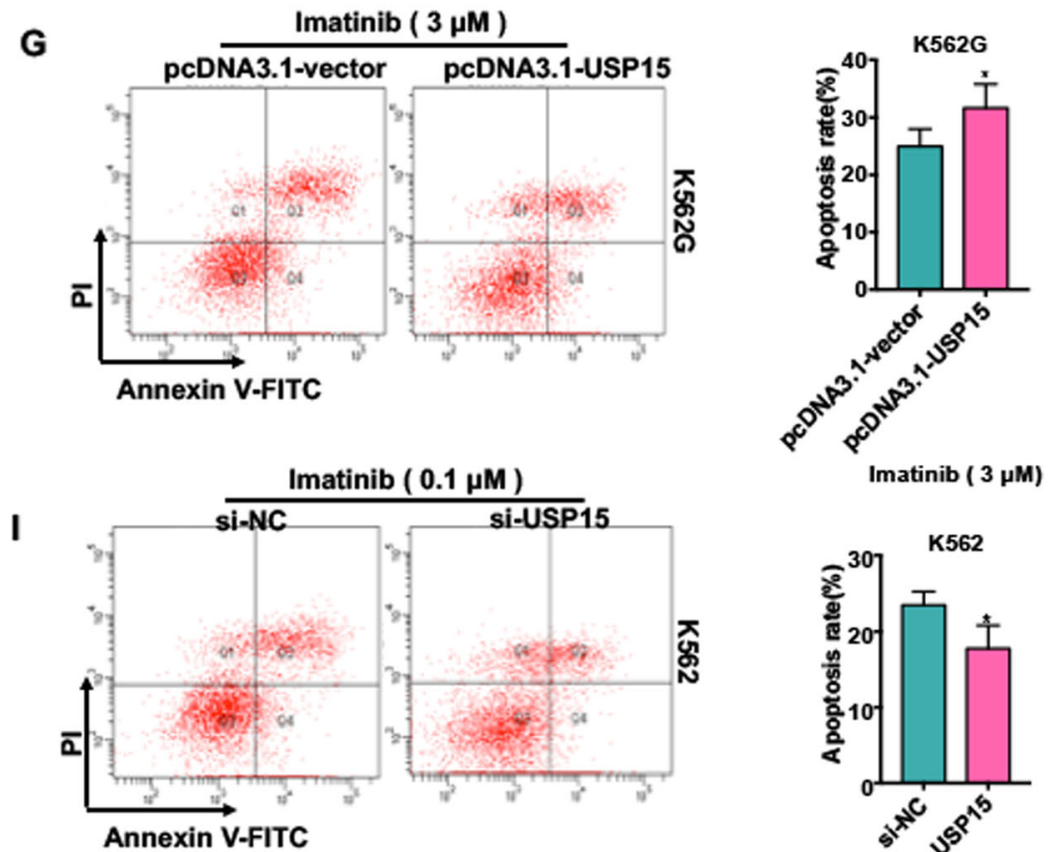

Imatinib ( $3 \mu \mathrm{M})$

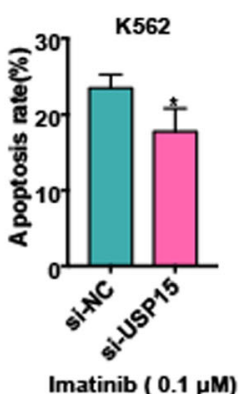

Fig. 2 Downregulation of USP15 contributes to Imatinib resistance of CML cells. (a) qRT-PCR was used to detect USP15 mRNA level in K562 cells, K562G cells and PBMCs of healthy donors. ${ }^{* *} P<0.001$ vs normal; \#\# $P<0.01$ vs. K562 cell. (b) Western blot analysis was used to evaluate USP15 protein level in K562 cells, K562G cells and PBMCs of healthy donor. (c) Immunofluorescence analyzed the USP15 protein level and the localization of USP15 in K562 cells, K562G cells and PBMCs of healthy donor. Scale bar $=64 \mu \mathrm{m}$. (d) Western blot analysis was used to evaluate USP15 protein level in different phases of CML (CML-CP, CML-BP-AML, CML-BP-ALL) patients' PBMCs compared with the PBMCs of normal donors. (e) qRT-PCR was used to test USP15 mRNA level in PBMCs of CML-CP $(n=6)$ patients and PBMCs of CML-BP patients $(n=6)$. ${ }^{*} P<0.05$ vs. CML-CP. (f) K562G cells were transfected with pcDNA3.1-USP15 or empty and then treated with Imatinib in different concentrations for $48 \mathrm{~h}$. Imatinib IC50 was evaluated by CCK-8 assay. ${ }^{*} P<0.01$ vs. pcDNA3.1-vector. (g) K562G cells were transfected with pcDNA3.1-USP15 or empty vector and then treated with Imatinib $(3 \mu \mathrm{m})$ for $48 \mathrm{~h}$. Cell apoptosis was evaluated by Annexin V-FITC/PI staining. Right panel shows the analysis of apoptosis rate. ${ }^{*} P<0.05$ vs. pcDNA3.1-vector. (h) K562 cells were transfected with si-USP15 or si-NC and then treated Imatinib in different concentrations for 48 h. Imatinib IC50 was evaluated by CCK-8 assay. ${ }^{* *} P<0.001$ vs. si-NC. (i) K562 cells were transfected with si-USP15 or si-NC and then treated with Imatinib $(0.1 \mu \mathrm{m})$ for $48 \mathrm{~h}$. Cell apoptosis were evaluated by Annexin V-FITC/PI staining. ${ }^{*} P<0.05$ vs si-NC 


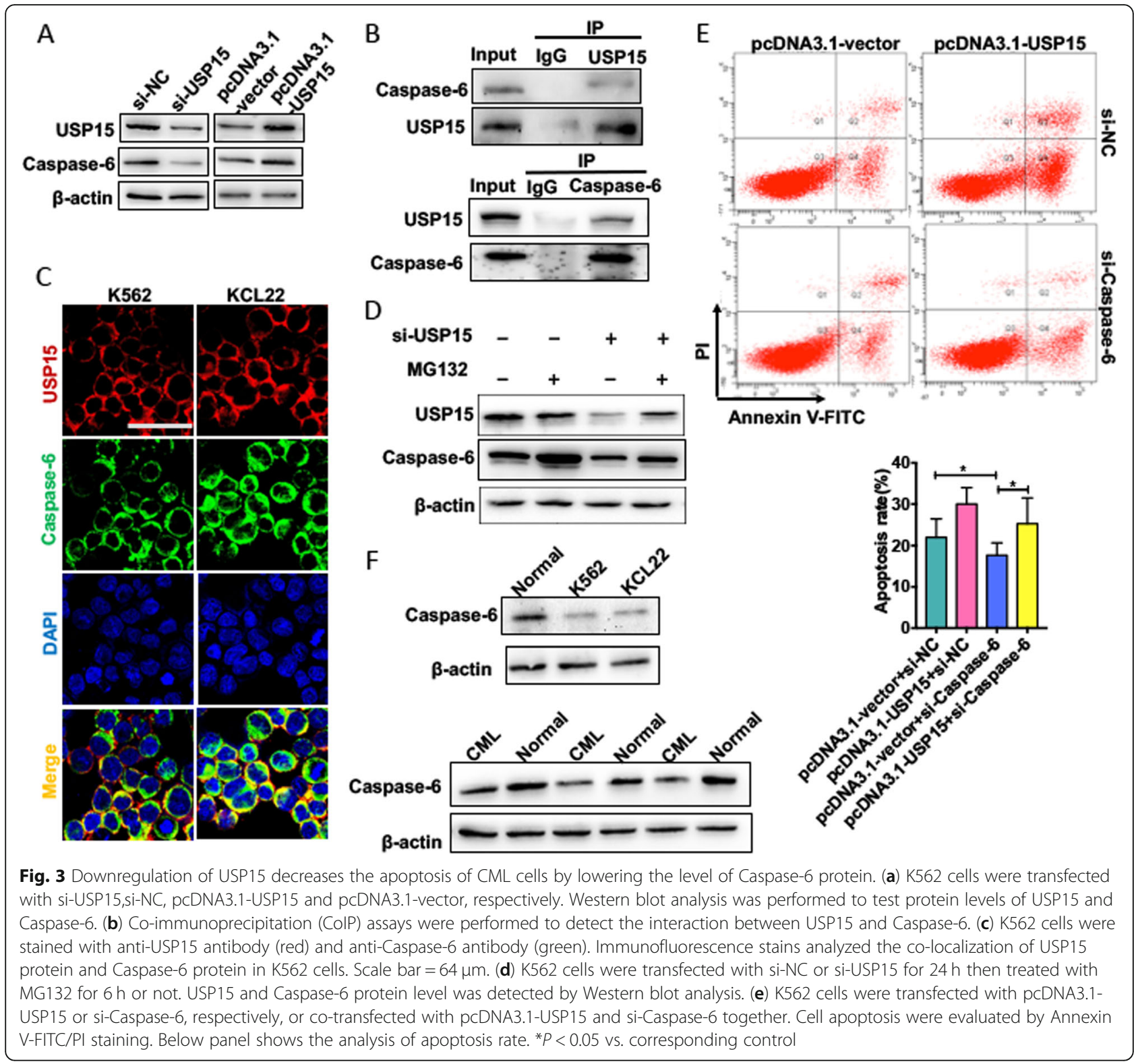

of Caspase-6. Thereafter, flow cytometry revealed that knockdown of Caspase- 6 decreased K562 cell apoptosis, and this effect could be reversed by overexpressing USP 15 (Fig. 3 e). In addition, the expression level of Caspase-6 protein was significantly decreased in CML cell line and CML patients (Fig. $3 \mathrm{f}$ ). These findings indicate that expression level of Caspase- 6 , as a downstream protein of USP15, is regulated at the post-translational level by deubiquitinating activity of USP15 and that downregulation of USP15 leads to decreased Caspase-6 level.

\section{USP15 is a direct target of miR-202-5p}

Because we found that USP15 downregulation led to decreased apoptosis in CML cells by lowering the level of Caspase- 6 protein, we sought to know whether the expression of USP15 is suppressed in CML cells by a microRNA that targets 3 '-untranslated region (3'-UTR) of USP15 mRNA. To do this, high-throughput sequencing analysis of microRNAs was performed in $\mathrm{K} 562 \mathrm{G}$ cells versus its parental cells. The results showed that 9 and 11 miRNAs were significantly upregulated and downregulated, respectively, in K562G cells (Fig. 4 a). miRNA target predictions by TargetScan (http://www. targetscan.org/vert_72/) were used to screen putative miRNAs that may target USP15 3'-UTR. Notably, miR202-5p was upregulated in K562G cells, and 3'-UTR of USP15 mRNA contains highly conserved miR-202-5pbinding site (Fig. 4 b). To determine whether miR-202$5 p$ regulates USP15 expression in CML cells, we transfected K562 cells with miR-202-5p mimic/inhibitor to 


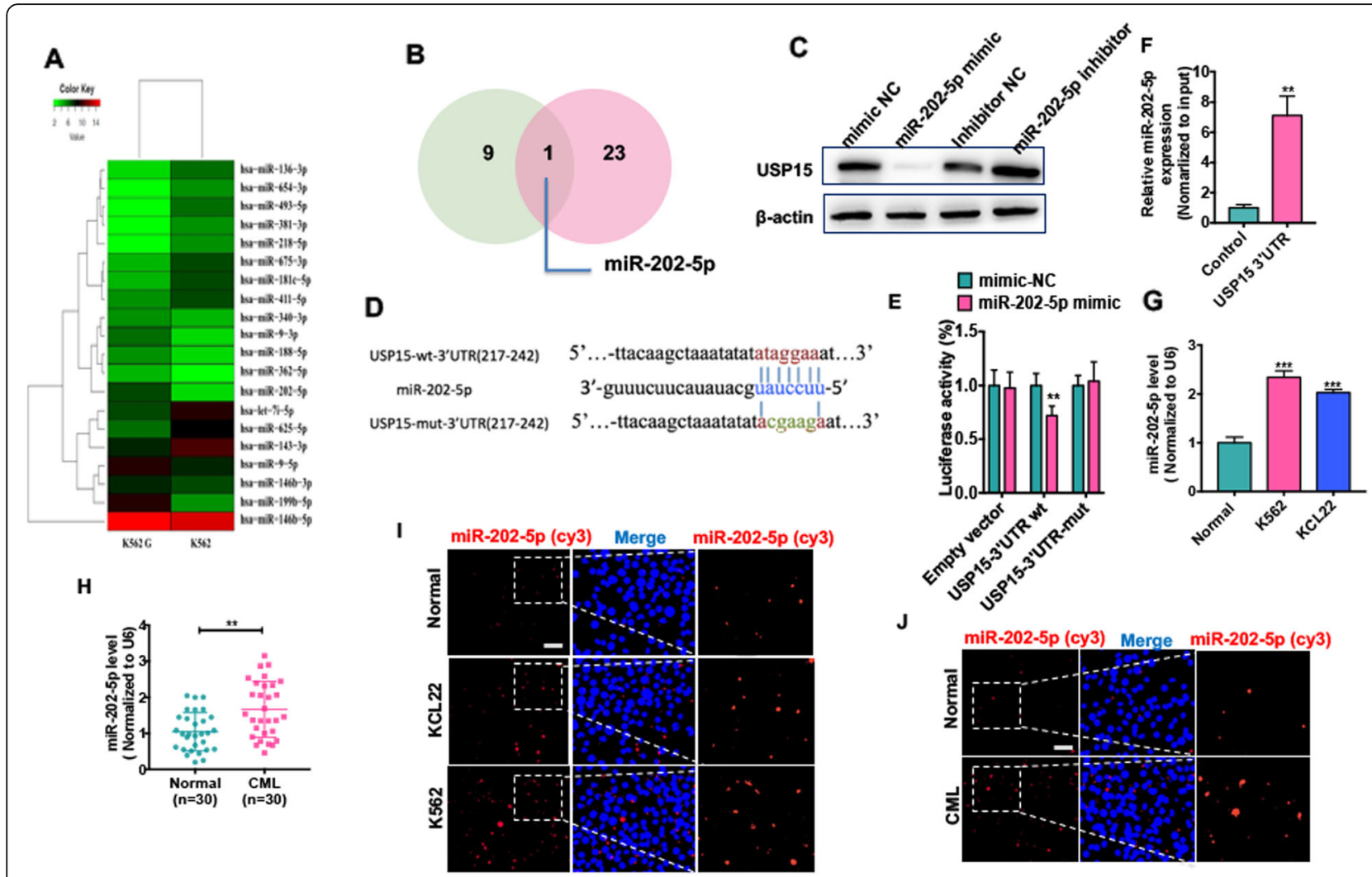

Fig. 4 USP15 as a direct target of miR-202-5p. (a) Heat map showing the differential expression (fold changes) of miRNA between K562 and K562G cells from RNA-seq analysis. (b) Venn diagram performed that miRNA upregulation in K562G (green panel) cells overlapped the predicted miRNA ((http://www.targetscan.org/vert_72/) (red panel) which may target USP15. (c) K562 cells were transfected with miR-202-5p mimic, mimicNC, miR-202-5p inhibitor or inhibitor-NC, respectively. Protein level of USP15 was measured by Western blot analysis. (d) Potential binding site of miR-202-5p at the 3' UTR of USP15. (e) K562 cells co-transfected cells with miR-202-5p mimic and wild-type (WT) or mutant (mut) USP15 3'-UTRluciferase reporter. Luciferase reporter assays were used to detect luciferase activity. ${ }^{* *} P<0.01$ vs. mimic-NC. (f) USP15 $3^{\prime}$-UTR and control RNA with biotin-labeled uridine triphosphate were transfected into $\mathrm{K} 562$ cells for $24 \mathrm{~h}$. The miRNAs were extracted after a pull-down assay, and miR202-5p expression was detected by qRT-PCR. Multiple miRNAs could be pulled down by RBM5 $3^{\prime}-$ UTR. **P $<0.01$ vs. control RNA. (g) qRT-PCR was used to test miR-202-5p level in CML cell lines (K562 and KCL22) and PBMCs of healthy donors. Normalized to U6. ${ }^{* * *} P<0.001$ vs. normal (h) qRTPCR detected the expression of miR-202-5p expression in PBMCs of CML-CP $(n=30)$ patients compared with PBMCs of healthy honors $(n=30)$. ${ }^{*} P<0.01$ vs. normal. (i) Fluorescence in situ hybridization (FISH) detected the miR-202-5p in CML cell lines and PBMCs of healthy donors. Blue staining represents the nucleus and red staining indicates miR-202-5p. Scale bar $=20 \mu \mathrm{m}$. (j) FISH detected the miR-202-5p in PBMCs of CML-CP patients and PBMCs of healthy donors. Scale bar $=20 \mu \mathrm{m}$

overexpress/knock down miR-202-5p. qRT-PCR results showed that miR-202-5p mimic significantly increased, while its inhibitor obviously reduced miR-202-5p expression level in K562 cells (Additional file 1: Fig. S5). Using loss- and gain-of-function experiments, we found that miR-202-5p mimic dramatically decreased, and miR202-5p inhibitor markedly increased USP15 protein level (Fig. 4 c). In further experiments, we used luciferase reporter assay to detect the effect of miR-202-5p on USP15 expression. As indicated in Fig. 4 d, there existed a putative binding site of miR-202-5p in 3'-UTR of USP15 mRNA. Transfecting K562 cells with miR-202-5p mimic, but not mimic-NC, obviously decreased luciferase activity driven by the wild type of USP15 3'-UTR. However, luciferase activity directed by the mutant-type of USP15 3'-UTR was not affected by miR-202-5p mimic (Fig. 4 e). Then we used a T7 RNA transcriptase to generate USP15 3'-UTR in vitro with biotin-labeled uracil. The USP15 3'-UTR was transfected into K562 cells, and miR-202-5p was enriched via the pull-down method. As shown in Fig. 5 f, miR-202-5p was significantly enriched in the precipitates by qRT-PCR, indicating that miR-202-5p could bind to USP15 3'UTR and regulate USP15 expression.

To investigate the clinical significance of miR-202-5p, we analyzed the expression level of miR-202-5p in CML patients and CML cell lines. The results showed that miR202-5p expression was much higher in K562 and KCL22 cell lines than in PBMCs of healthy donors (Fig. 4 g). This was also confirmed in PBMCs of CML-CP patients by qRT-PCR (Fig. 4 h). Fluorescent in situ hybridization (FISH) analysis showed that miR-202-5p obviously 


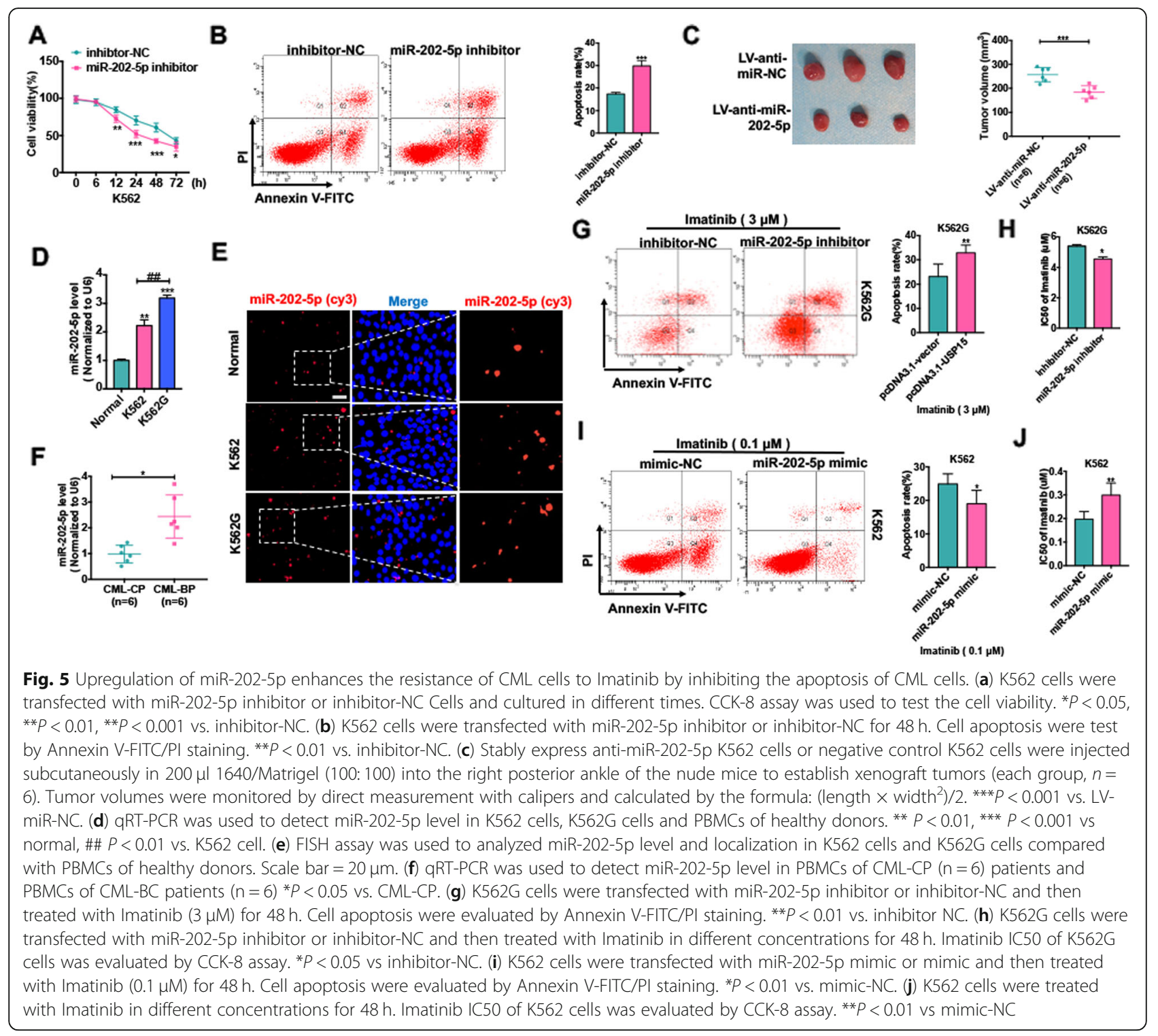

increased in CML cell lines and PBMCs of CML patients and was localized in the cytoplasm of these cells (Fig. 4 i and j).

\section{Upregulation of miR-202-5p enhances the resistance of CML cells to Imatinib by inhibiting the apoptosis of CML cells}

Considering that miR-202-5p is increased in CML cells, its impact on CML cell proliferation and apoptosis was evaluated. We knocked down miR-202-5p in K562 cells by miR-202-5p inhibitor and found that depletion of miR-202-5p dramatically inhibited cell proliferation and increased apoptosis compared with those transfected with inhibitor-NC (Fig. $5 \mathrm{a}$ and b). Further, the K562 cells stably expressing anti-miR-202-5p were implanted into nude mice to observe the effect of miR-202-5p depletion on K562 cell xenograft growth. As a result, tumor volume was significantly reduced in miR-202-5pdepleted mice by LV-anti-miR-202-5p compared with control mice (Fig. 5 c). These findings indicate that upregulation of miR-202-5p results in an increased proliferation and decreased apoptosis in CML cells.

To further clarify whether Imatinib resistance of CML cells is related to miR-202-5p upregulation, we detected the expression level of miR-202-5p in K562G and K562 cells. qRT-PCR and FISH analysis showed that miR-202$5 \mathrm{p}$ expression level was much higher in K562G cells than in K562 cells (Fig. $5 \mathrm{~d}$ and e). Similarly, miR202-5p level was also significantly upregulated in CML-BC patients compared with that in CML-CP patients (Fig. 5 f). Next, we depleted miR-202-5p in K562G cells by miR-202-5p inhibitor and found that 
depletion of miR-202-5p decreased the IC50 values of Imatinib, thus facilitating Imatinib-induced apoptosis of K562G cells (Fig. $5 \mathrm{~g}$ and h). In contrast, transfection of K562 cells with miR-202-5p mimic increased the IC50 values of Imatinib and inhibited K562 cell apoptosis induced by Imatinib (Fig. $5 \mathrm{i}$ and j). These findings indicate that upregulation of miR-202-5p enhances the resistance of CML cells to Imatinib by inhibiting cell apoptosis.

STAT5A is upregulated in CML cells and directly activates miR-202-5p transcription by binding to the pre-miR-202 promoter

To investigate the mechanism underlying upregulation of miR-202-5p in CML cells, we used an online software PROMO (http://alggen.lsi.upc.es) to predict the putative transcriptional factors that can bind to the pre-miR-202 promoter. The results revealed that STAT5A, WT1 [21] and CEBPA [22], all of which can be activated by BCR$\mathrm{ABL}$, were predicted to be capable of binding to the premiR-202 promoter. Next, we examined the effect of these three transcriptional factors on pre-miR-202-5p expression. As shown in Additional file 1: Fig. S6, knockdown of STAT5A by its specific si-RNA significantly decreased pre-miR-202-5p level in K562 cells, however, knockdown of CEBPA and WT1 did not affect pre-miR202-5p expression. Thus, we focused our attention on STAT5A in the subsequent experiments. First, we demonstrated that overexpression or knockdown of STAT5A in K562 cells significantly increased or decreased both total STAT5 and p-STAT5A level (Additional file 1: Fig. S7). Then, qRT-PCR results showed that overexpression or knockdown of STAT5A also significantly upregulated or suppressed, respectively, miR-202-5p expression compared with their corresponding control (Fig. 6 a and b). To provide additional confirmation that STAT5A directly activates pre-miR-202-5p transcription, we used PROMO prediction software to identify the putative binding sites of STAT5A in the pre-miR-202 promoter and found that 3 putative binding sites existed in the pre-miR-202 promoter region. ChIP-PCR analysis showed that STAT5A was directly bound to the -4 to $-256 \mathrm{bp}$ and the -588 to $-821 \mathrm{bp}$ region of the premiR-202 promoter (Fig. 6 c). Furthermore, luciferase activity assay revealed that co-transfecting $\mathrm{K} 562$ cells with STAT5A expression vector and pre-miR-202 promoterdirected luciferase reporter significantly increased luciferase activity compared with that transfected with empty vector (Fig. 6 d).

In the further experiments, we determined whether STAT5A affects the expression of USP15 and Caspase-6. The results showed that enforced STAT5A expression in K562 cells markedly attenuated the expression level of USP15 and Caspase-6, whereas knockdown of STAT5A had the opposite effects (Fig. 6 e). Additionally, we found that depletion of endogens miR-202-5p by miR-202-5p inhibitor could abrogate the inhibitory effect of STAT5A overexpression on USP15 and Caspase- 6 expression (Fig. $6 \mathrm{f}$ ), indicating that miR-202-5p mediates STAT5A repression of USP15 expression. Additionally, CML patients and cell lines had a lower mRNA expression level than the healthy PBMCs (Fig. $6 \mathrm{~g}$ and h). Protein level of STAT5A and p-STAT5A was also increased in CML patients and cell lines detected by Western blotting (Fig. 6 $i$ and $j)$. These findings suggest that STAT5A positively regulates miR-202-5p expression by binding to the promoter of the miR-202-5p precursor.

\section{Pimozide induces CML cell apoptosis by blocking STAT5A/miR-202-5p/USP15/Caspase-6 regulatory pathway} Previous study showed that pimozide, a neuroleptic drug, potently induces apoptosis in CML cells by inhibiting STAT5 activity [23]. In this study, our results indicated that STAT5A upregulation in CML cells facilitates the formation of miR-202-5p/USP15/ Caspase-6 regulatory axis, thus suppressing the apoptosis of CML cells. Here, we sought to determine whether pimozide promotes CML cell apoptosis by regulating STAT5/miR-202-5p/USP15/Caspase-6 axis. First, we showed that the level of phosphorylated STAT5A (p-STAT5A) was remarkedly reduced in pimozide-treated $\mathrm{K} 562$ cells without affecting the total STAT5 protein level (Fig. 7 a). Further, treating K562 cells with pimozide also significantly decreased miR202-5p expression level compared with DMSO control (Fig. 7 b). Correspondingly, pimozide enhanced the expression level of USP15 and Caspase- 6 protein, as shown by Western blotting (Fig. 7 c), and miR-202$5 \mathrm{p}$ mimic abolished the upregulation of USP15 and Caspase- 6 induced by pimozide (Fig. 7 d), indicating that pimozide can block STAT5A/miR-202-5p/USP15/ Caspase- 6 regulatory pathway via inhibiting the phosphorylation of STAT5A.

Next, the effect of pimozide on CML cell apoptosis was evaluated. As expected, pimozide significantly induced K562 cell apoptosis compared with DMSO control (Fig. 7 e). More importantly, treating K562G cells with Imatinib combined with pimozide could obviously increase the apoptosis rate of $\mathrm{K} 562 \mathrm{G}$ cells compared with Imatinib alone (Fig. 7 f). Finally, in vivo experiments showed that pimozide dramatically reduced tumor volume in K562 cell xenograft model (Fig. 7 g). At the same time, we also detected miR-202-5p expression in the xenograft tumors. The results showed that pimozide significantly decreased the expression level of miR-202-5p compared with control group (Fig. 7 h). Similarly, in K562 cell xenograft tumors, pimozide also 


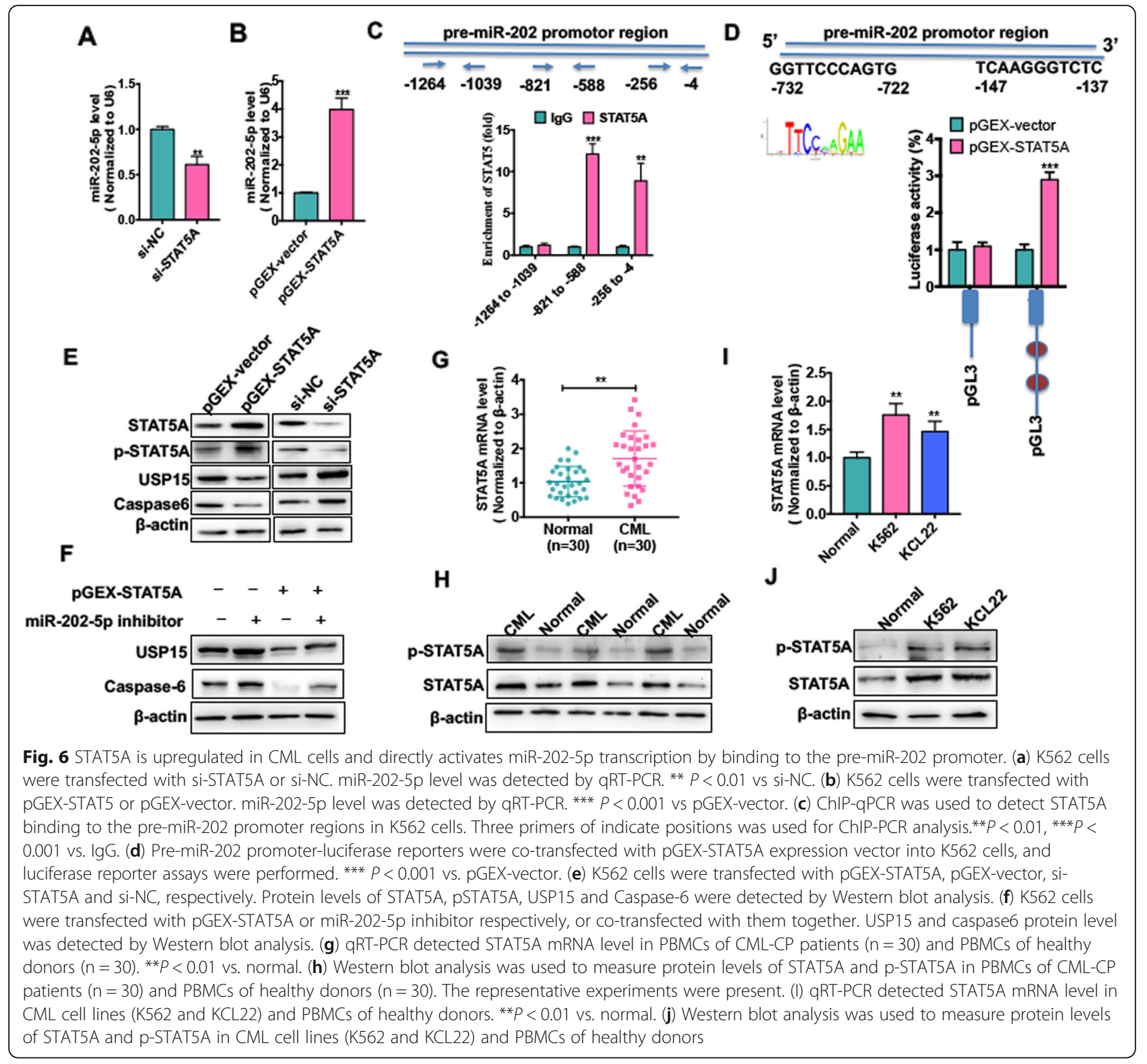

obviously suppressed STAT5A phosphorylation and markedly enhanced the expression of USP15 and Caspase-6, thus facilitating CML cell apoptosis (Fig. 7 i). These data again suggest that pimozide exerts its antileukemia effects by inhibiting STAT5A phosphorylation and thus blocking STAT5A/miR-202-5p/USP15/Caspase- 6 regulatory pathway. As proposal model shown in Fig. 8, we found dysregulation of STAT5A/miR-202-5p/ USP15/Caspase- 6 regulatory axis contributed to CML cell apoptosis and Imatinib resistance.

\section{Discussion}

Imatinib mesylate, the first tyrosine kinase inhibitors (TKIs) targeting BCR-ABL1, have effectively improved the survival of patients with CML [24]. However, 20-
$30 \%$ of CML patients haven't been benefited from TKI treatment commonly due to TKIs resistance which lead to disease relapse and progression [25]. Most resistance of TKIs can be mediated by mutations within BCR-ABL kinase domain that interfere with ATP binding site [26]. However, approximately 50\% Imatinib-resistant CML patients has not mutations in ABL domain. Therefore, BCR-ABL-independent Imatinib-resistant mechanisms may also play a role in progressive disease [27]. Previously studies have reported that the abnormal activation of pathway or gene expression may be associated with Imatinib resistance. For example, Moshe showed that LYN kinase was overexpressed and activation in Imatinib-resistant $\mathrm{K} 562$ cells, suggesting that expression or activation of a SRC-family kinase played a role in 


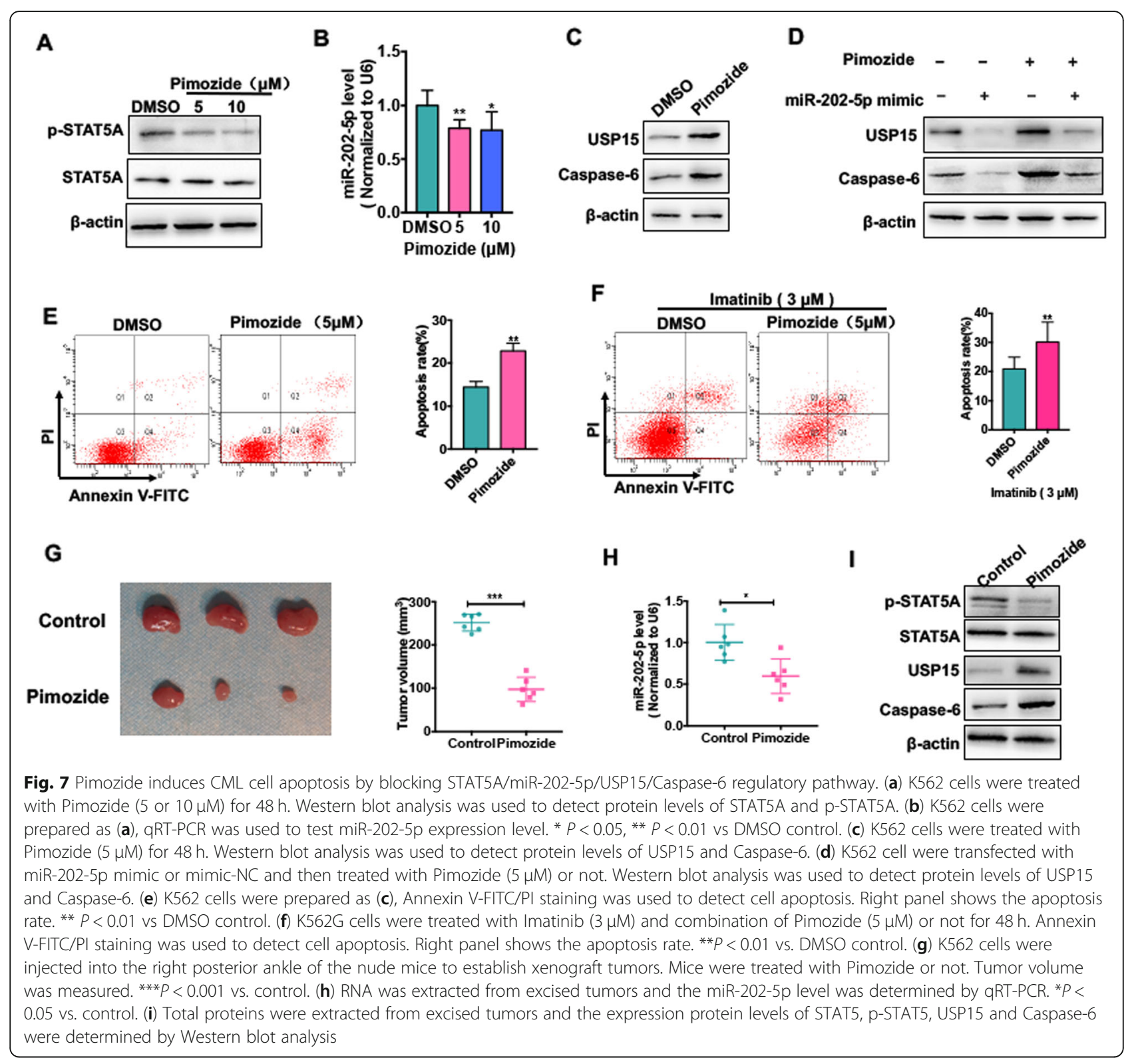

Imatinib resistance. Liu revealed TKI-resistant CML primary cells and cell line had a higher level of PKM2 level. Knockdown of PKM2 expression reduced cell proliferation, and induced cell apoptosis of TKI-resistant cell line after treated with Imatinib by regulation of glucose metabolism [28]. Besides, Salvatore revealed 24 proteins were overexpressed in Imatinib-resistant KCL22 compared with its parental cells and some of these proteins were involved cell-cycle pathway and anti-apoptotic pathways. In the present study, we first confirmed that USP15 is downregulated in CML cell lines and PBMCs of patients with CML-CP. The in vivo and in vitro experiments revealed overexpression of USP15 significantly reduced the cell viability and thus induced the apoptosis of $\mathrm{K} 562$ cells. Furthermore, Imatinib resistant K562G cells and patients with Imatinib resistance had much lower expression level of USP15. Depletion of USP15 increased, while overexpression of USP15 reduced the resistance of CML cells to Imatinib, suggesting that USP15 may function as a tumor suppressor in CML and involve in Imatinib resistance.

The ubiquitin-proteasome pathway is a critical mechanism for regulating the protein degradation [29]. Deubiquitinases (DUBs) are a group of enzymes that could remove ubiquitin chains from ubiquitinated proteins, leading to prevention the target proteins from degradation [30]. The ubiquitin-specific peptidases (USPs) are the main members of the deubiquitinase family. Increasing evidence have indicated that dysregulation of USPs contributes to multiple tumor progressions, including 


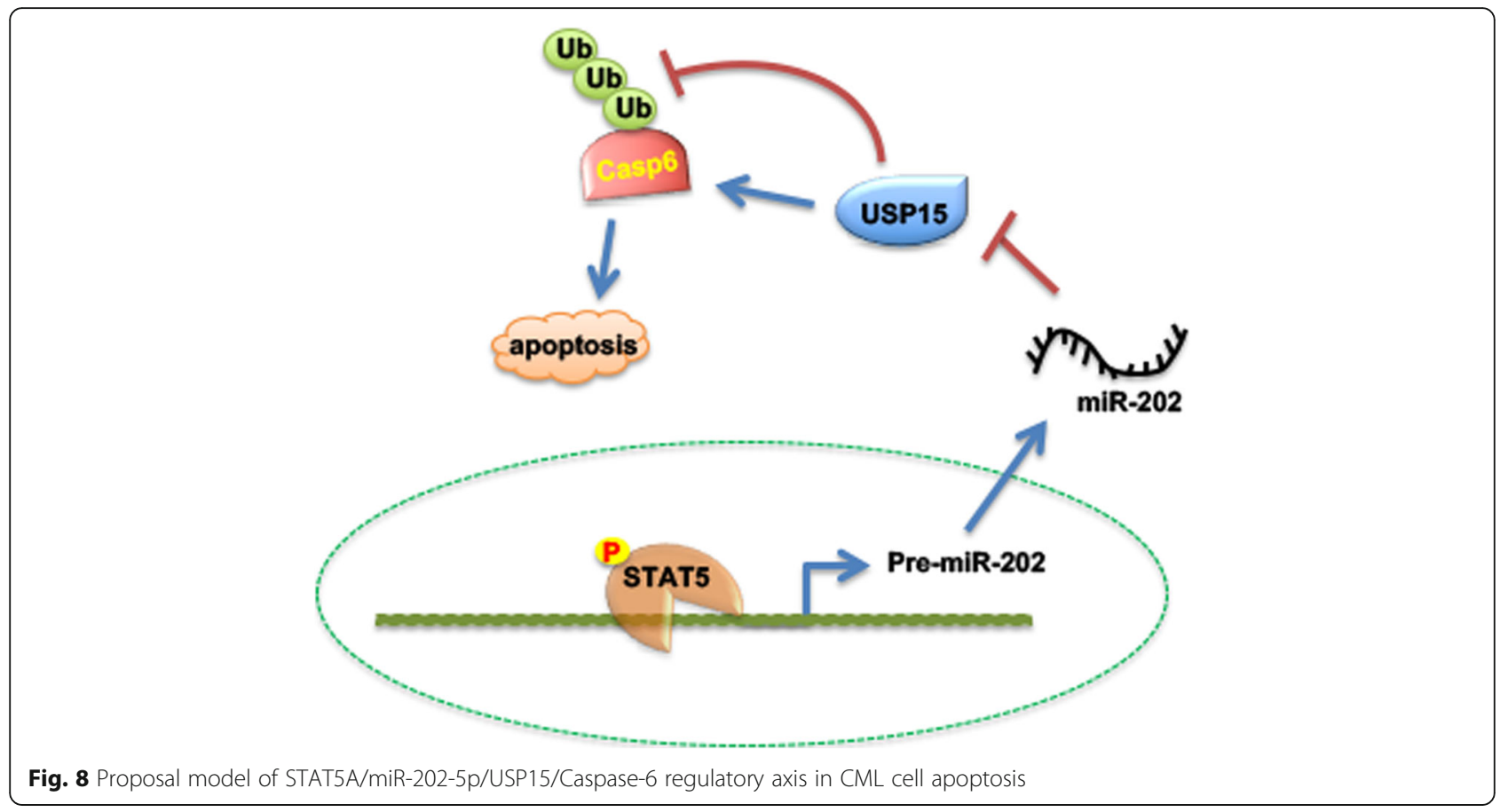

hematological malignancies [31]. For example, Kitamura showed the functional role of USP7 in the deubiquitination and stabilization of ASXL1 which is an independent poor prognostic factor among patients with MDS. Depletion of USP7 in HL-60 cells decreased ASXL1 protein level, suggesting that USP7 inhibitor may a potential target in AML and MDS treatment [32]. Jin reported that USP7 was overexpressed in T-ALL cells and suppression of USP7 led to significantly decrease T-ALL cell growth in vitro and in vivo by controlling the $\mathrm{NOTCH} 1$ protein level through deubiquitination [33]. Liu found that USP10 acted as the DUBs of SKP2 which may also promote leukemogenesis and stabilize SKP2 protein in CML cells. The in vivo and in vitro experiments confirmed that inhibition of USP10 suppressed the proliferation of CML [34]. In the present study, we found that 25 proteins might be interacted with USP15 analyzed by CoIP with mass spectrometry analysis. We further demonstrated Caspase-6, as a downstream protein of USP15, was regulated at the post-translational level by deubiquitinating activity of USP15. Caspase-6 protein level was positively regulated by USP 15 and attributed to the apoptosis induced by USP15 in CML cells. However, as the deubiquitinases, USP15 may regulate more proteins degradation by deubiquitination. Further researches need to clarify the more downstream gene of USP15 and their biological functions.

These results raise the important questions about how USP15 expression is regulated in CML cells. Mechanisms controlling genes expression include transcript regulation, mutations of gene, aberrations in gene copy number, epigenetic mechanisms and micro-RNA (miRNA) regulation. miRNAs are small non-coding RNAs which negatively regulate gene expression in posttranslational level through the degradation of transcripts or by interfering with protein translation [35]. It is widely accepted that abnormal expression of miRNAs may be responsible for the tumor development by targeting oncogene or tumor suppressor [36], including USPs. For example, in melanoma, overexpression of miR-593-3p led to the decrease USP13 level involved in melanoma metastasis [37]. In endocrine-resistant breast cancer, USP19 regulated by miR-15a may response to the drug resistance. In osteosarcoma, USP1 level was upregulated in osteosarcoma tissues and cell lines and due to the regulation of miR-192-5p [38]. In colorectal cancer, USP 13 targeted by miR-224 and indirectly regulated smad4 gene level in colorectal cancer cells [39]. In the present study, we revealed that miR-202-5p which was overexpression in CML cell lines and patients directly targeted USP15 3'UTR and negatively regulated USP15 protein level in CML cells. Subsequently, miR-202-5p as an onco-miRNA, was responsible to CML cell proliferation and apoptosis. Importantly, miR-202-5p was higher in Imatinib-resistant CML cells and played a role in Imatinib-resistance, suggesting that miR-202-5p might be a potential biomarker in Imatinib resistance. Nevertheless, one miRNA may suppress multiple gene expression, while altered gene expression may also be affected by a variety of factors. In the present study, we found a novel mechanism for USP15 depletion in CML and Imatinib resistance was due to the overexpression of miR- 
202-5p. Other unknown regulations of USP15 depletion in $\mathrm{CML}$ requires more in vivo and in vitro experiments.

In the present study, we further demonstrated that the STAT5A crosslinked into pre-miR-202 promotor element and transcriptional up-regulated miR-202-5p expression, as evidenced by luciferase reporter gene assays and ChIP analysis. It has been clearly known that STAT5A is a master downstream effector of BCR-ABL [40]. Altered activation of STAT5A which affected by BCR-ABL tyrosine kinase can influence the CML development and progression [41]. Consistent with previously described [42], we found significantly increased expression level of STAT5 in CML cell lines and patients, especially in Imatinib-resistant patients and CML cell line. Besides, the new downstream axis of STAT5A was explored in the study. Depletion of STAT5A could dramatically increase USP15 and Caspase-6 expression levels in CML cells mediating by miR-202-5p and associated with CML apoptosis and IM resistance. That is to say, initiating from abnormal expression and activation of STAT5A, dysregulation of STAT5A/miR-202-5p/USP15/Caspase-6 regulatory axis exerts an critical role in CML cells apoptosis Imatinib resistance. Importantly, we proved that the Pimozide, as the STAT5A inhibitor, could effectively reduce the phosphorylation of STAT5A thus inhibit the activation of STAT5A/miR-202-5p/USP15/Caspase-6 regulatory axis pathway. Stimulation of Pimozide in CML cells could obviously increase USP15 and Caspase6 expression levels, indicating that Pimozide functioned as USP15 activator though suppressing miR-202-5p level. Furthermore, our findings substantiated that Pimozide promoted CML cell apoptosis and inhibited cell growth in vivo and in vitro, consistent with the previously report [23, 43]. Subsequently, Pimozide enhanced Imatinib sensitivity in Imatinib resistant K562 cells, suggesting Pimozide may be a promising adjuvant to treat patients with CML.

\section{Conclusion}

In conclusion, as proposal model shown in Fig. 8, we found dysregulation of STAT5A/miR-202-5p/USP15/ Caspase- 6 regulatory axis contributed to CML cell apoptosis and Imatinib resistance. Hence, targeting this pathway may can be a novel treatment for CML and overcoming Imatinib resistance.

\section{Additional files}

Additional file 1. Supplementary Figures and Tables.

Additional file 2. Supplemental Experimental Procedures.

\section{Abbreviations}

ALL: Acute lymphoblastic leukemia;; ChIP: Chromatin immunoprecipitation;" CML: Chronic myeloid leukemia; ColP: Co-immunoprecipitation;"
DUBs: Deubiquitinases;; PI3K: Phosphoinositide 3-kinase; RT-qPCR: Reverse transcription-quantitative polymerase chain reaction; transducer and activator of transcription 5 ; TCGA: The Cancer Genome Atlas;" TKIs: Tyrosine kinase inhibitors; USPs: Ubiquitin-specific peptidases;"; UTR: untranslated region;

\section{Acknowledgements \\ We like to thank Prof. Jin-Kun Wen and Prof. Bin Zheng for technology supports.}

\section{Authors' contributions}

Conception and design: J.L.,L.J and Z.N; Tissues collection: Z.Y., M.Y. and N.Z;; Development of methodology: Z.N., M.L.,X.Z. and X.L.; Acquisition of the data: L.Y., Z.N. and Z.Y.; Analysis of data: Z.N.,M.Y. and J.L.; Writing the manuscript: Z.N.,L.Y. and M.Y. All authors read and approved the final manuscript.

\section{Funding}

Not applicable.

\section{Availability of data and materials}

Not applicable.

\section{Ethics approval and consent to participate}

The present study was authorized Ethics Committee of Second Hospital of Hebei Medical University. All patients and volunteers were anonymous and provided written informed consent.

\section{Consent for publication}

Written consent was obtained from all participants.

\section{Competing interests}

The authors declare that they have no competing interests.

\section{Author details}

${ }^{1}$ Department of Hematology, The Second Hospital of Hebei Medical University, 215 Heping W Rd, Shijiazhuang 050000, China. ${ }^{2}$ Department of Biochemistry and Molecular Biology, The Key Laboratory of Neural and Vascular Biology, Ministry of Education of China, Hebei Medical University, No. 361 Zhongshan E Rd, Shijiazhuang 050017, China. ${ }^{3}$ Department of Urology, The Second Hospital of Hebei Medical University, 215 Heping W Rd, Shijiazhuang 050000, China.

Received: 8 September 2019 Accepted: 12 December 2019

Published online: 17 January 2020

\section{References}

1. Nash I. Chronic myeloid leukemia. N Engl J Med. 1999;341(10):765.

2. Deininger MW, Goldman JM, Melo JV. The molecular biology of chronic myeloid leukemia. Blood. 2000;96(10):3343-56.

3. Cortez D, Stoica G, Pierce JH, Pendergast AM. The BCR-ABL tyrosine kinase inhibits apoptosis by activating a Ras-dependent signaling pathway. Oncogene. 1996;13(12):2589-94.

4. Steelman LS, Pohnert SC, Shelton JG, Franklin RA, Bertrand FE, McCubrey JA. JAK/STAT, Raf/MEKJERK, PI3K/Akt and BCR-ABL in cell cycle progression and leukemogenesis. Leukemia. 2004;18(2):189-218.

5. Liu WT, Huang KY, Lu MC, Huang HL, Chen CY, Cheng YL, Yu HC, Liu SQ, Lai NS, Huang HB. TGF-beta upregulates the translation of USP15 via the PI3K AKT pathway to promote p53 stability. Oncogene. 2017;36(19):2715-23.

6. Eichhorn PJ, Rodon L, Gonzalez-Junca A, Dirac A, Gili M, Martinez-Saez E, Aura C, Barba I, Peg V, Prat A, et al. USP15 stabilizes TGF-beta receptor I and promotes oncogenesis through the activation of TGF-beta signaling in glioblastoma. Nat Med. 2012;18(3):429-35.

7. Padmanabhan A, Candelaria N, Wong KK, Nikolai BC, Lonard DM, O'Malley BW, Richards JS. USP15-dependent lysosomal pathway controls p53-R175H turnover in ovarian cancer cells. Nat Commun. 2018;9(1):1270.

8. Zhou L, Jiang H, Du J, Li L, Li R, Lu J, Fu W, Hou J. USP15 inhibits multiple myeloma cell apoptosis through activating a feedback loop with the transcription factor NF-kappaBp65. Exp Mol Med. 2018;50(11):151.

9. Xu M, Takanashi M, Oikawa K, Tanaka M, Nishi H, Isaka K, Kudo M, Kuroda M. USP15 plays an essential role for caspase-3 activation during paclitaxelinduced apoptosis. Biochem Biophys Res Commun. 2009;388(2):366-71. 
10. Zou Q, Jin J, Hu H, Li HS, Romano S, Xiao Y, Nakaya M, Zhou X, Cheng X, Yang $P$, et al. USP15 stabilizes MDM2 to mediate cancer-cell survival and inhibit antitumor T cell responses. Nat Immunol. 2014;15(6):562-70.

11. Huang X, Langelotz C, Hetfeld-Pechoc BK, Schwenk W, Dubiel W. The COP9 signalosome mediates beta-catenin degradation by deneddylation and blocks adenomatous polyposis coli destruction via USP15. J Mol Biol. 2009; 391(4):691-702.

12. Basham B, Sathe M, Grein J, McClanahan T, D'Andrea A, Lees E, Rascle A. In vivo identification of novel STAT5 target genes. Nucleic Acids Res. 2008; 36(11):3802-18.

13. Turhan AG. STAT5 as a CML target: STATinib therapies? Blood. 2011;117(12): 3252-3.

14. Sibbesen NA, Kopp KL, Litvinov IV, Jonson L, Willerslev-Olsen A, Fredholm S, Petersen DL, Nastasi C, Krejsgaard T, Lindahl LM, et al. Jak3, STAT3, and STAT5 inhibit expression of miR-22, a novel tumor suppressor microRNA, in cutaneous T-cell lymphoma. Oncotarget. 2015;6(24):20555-69.

15. Haetscher N, Feuermann Y, Wingert S, Rehage M, Thalheimer FB, Weiser C, Bohnenberger $H$, Jung K, Schroeder T, Serve H, et al. STAT5-regulated microRNA-193b controls haematopoietic stem and progenitor cell expansion by modulating cytokine receptor signalling. Nat Commun. 2015; $6: 8928$.

16. Donato NJ, Wu JY, Stapley J, Gallick G, Lin H, Arlinghaus R, Talpaz M. BCR$A B L$ independence and $L Y N$ kinase overexpression in chronic myelogenous leukemia cells selected for resistance to STI571. Blood. 2003;101(2):690-8.

17. Chou CK, Chang YT, Korinek M, Chen YT, Yang YT, Leu S, Lin IL, Tang CJ, Chiu CC. The Regulations of Deubiquitinase USP15 and Its Pathophysiological Mechanisms in Diseases. Int J Mol Sci. 2017;18(3).

18. Villeneuve NF, Tian W, Wu T, Sun Z, Lau A, Chapman E, Fang D, Zhang DD USP15 negatively regulates Nrf2 through deubiquitination of Keap1. Mol Cell. 2013:51(1):68-79.

19. Baker RT, Wang XW, Woollatt E, White JA, Sutherland GR. Identification, functional characterization, and chromosomal localization of USP15, a novel human ubiquitin-specific protease related to the UNP oncoprotein, and a systematic nomenclature for human ubiquitin-specific proteases. Genomics. 1999;59(3):264-74.

20. Cowling V, Downward J. Caspase-6 is the direct activator of caspase-8 in the cytochrome c-induced apoptosis pathway: absolute requirement for removal of caspase-6 prodomain. Cell Death Differ. 2002;9(10):1046-56.

21. Zhang L, Li Y, Li X, Zhang Q, Qiu S, Zhang Q, Wang M, Xing H, Rao Q, Tian $Z$, et al. Regulation of HtrA2 on WT1 gene expression under imatinib stimulation and its effects on the cell biology of K562 cells. Oncol Lett. 2017;14(3):3862-8

22. Wagner K, Zhang P, Rosenbauer F, Drescher B, Kobayashi S, Radomska HS, Kutok JL, Gilliland DG, Krauter J, Tenen DG. Absence of the transcription factor CCAAT enhancer binding protein alpha results in loss of myeloid identity in bcr/abl-induced malignancy. Proc Natl Acad Sci U S A. 2006; 103(16):6338-43.

23. Nelson EA, Walker SR, Weisberg E, Bar-Natan M, Barrett R, Gashin LB, Terrell S, Klitgaard JL, Santo L, Addorio MR, et al. The STAT5 inhibitor pimozide decreases survival of chronic myelogenous leukemia cells resistant to kinase inhibitors. Blood. 2011;117(12):3421-9.

24. Castagnetti F, Gugliotta G, Breccia M, Stagno F, lurlo A, Albano F, Abruzzese E, Martino B, Levato L, Intermesoli T, et al. Long-term outcome of chronic myeloid leukemia patients treated frontline with imatinib. Leukemia. 2015; 29(9):1823-31.

25. Hochhaus A, Larson RA, Guilhot F, Radich JP, Branford S, Hughes TP, Baccarani M, Deininger MW, Cervantes F, Fujihara S, et al. Long-term outcomes of Imatinib treatment for chronic myeloid leukemia. N Engl J Med. 2017;376(10):917-27.

26. Jabbour E, Branford S, Saglio G, Jones D, Cortes JE, Kantarjian HM. Practical advice for determining the role of BCR-ABL mutations in guiding tyrosine kinase inhibitor therapy in patients with chronic myeloid leukemia. Cancer. 2011;117(9):1800-11

27. Flanigan DP, Williams LR. Venous insufficiency of the lower extremities: new methods of diagnosis and therapy. Surg Annu. 1982;14:359-80.

28. Tong L, Xu N, Zhou X, Huang JX, Wu WE, Chen C, Liang L, Liu QF, Liu XL. PKM2 mediates chronic myeloid leukemia Imatinib resistance by regulating glycolysis energy metabolism. Blood. 2018;132

29. Ciechanover A. The ubiquitin-proteasome proteolytic pathway. Cell. 1994; 79(1):13-21.
30. Komander D, Clague MJ, Urbe S. Breaking the chains: structure and function of the deubiquitinases. Nat Rev Mol Cell Biol. 2009;10(8):550-63.

31. Fraile JM, Quesada V, Rodriguez D, Freije JM, Lopez-Otin C. Deubiquitinases in cancer: new functions and therapeutic options. Oncogene. 2012;31(19): 2373-88.

32. Inoue D, Nishimura K, Kozuka-Hata H, Oyama M, Kitamura T. The stability of epigenetic factor ASXL1 is regulated through ubiquitination and USP7mediated deubiquitination. Leukemia. 2015;29(11):2257-60.

33. Jin Q, Martinez CA, Arcipowski KM, Zhu Y, Gutierrez-Diaz BT, Wang KK, Johnson MR, Volk AG, Wang F, Wu J, et al. USP7 cooperates with NOTCH1 to drive the oncogenic transcriptional program in T-cell leukemia. Clin Cancer Res. 2019;25(1):222-39.

34. Liao Y, Liu N, Xia X, Guo Z, Li Y, Jiang L, Zhou R, Tang D, Huang H, Liu J. USP10 modulates the SKP2/Bcr-Abl axis via stabilizing SKP2 in chronic myeloid leukemia. Cell Discov. 2019:5:24.

35. Bartel DP. MicroRNAs: target recognition and regulatory functions. Cell. 2009;136(2):215-33.

36. Chen CZ. MicroRNAs as oncogenes and tumor suppressors. New Engl J Med. 2005;353(17):1768-71.

37. Wang Y, Ou Z, Sun Y, Yeh S, Wang X, Long J, Chang C. Androgen receptor promotes melanoma metastasis via altering the miRNA-539-3p/USP13/MITF/ AXL signals. Oncogene. 2017;36(12):1644-54.

38. Zhou S, Xiong M, Dai G, Yu L, Zhang Z, Chen J, Guo W. MicroRNA-192-5p suppresses the initiation and progression of osteosarcoma by targeting USP1. Oncol Lett. 2018;15(5):6947-56.

39. Wang Z, Yang J, Di J, Cui M, Xing J, Wu F, Wu W, Yang H, Zhang C, Yao Z, et al. Downregulated USP3 mRNA functions as a competitive endogenous RNA of SMAD4 by sponging miR-224 and promotes metastasis in colorectal cancer. Sci Rep. 2017;7(1):4281.

40. Warsch W, Grundschober E, SexI V. Adding a new facet to STAT5 in CML: multitasking for leukemic cells. Cell Cycle. 2013;12(12):1813-4.

41. Warsch W, Kollmann K, Eckelhart E, Fajmann S, Cerny-Reiterer S, Holbl A Gleixner KV, Dworzak M, Mayerhofer M, Hoermann G, et al. High STAT5 levels mediate imatinib resistance and indicate disease progression in chronic myeloid leukemia. Blood. 2011;117(12):3409-20.

42. Cheng Y, Hao Y, Zhang A, Hu C, Jiang X, Wu Q, Xu X. Persistent STAT5mediated ROS production and involvement of aberrant p53 apoptotic signaling in the resistance of chronic myeloid leukemia to imatinib. Int $J$ Mol Med. 2018;41(1):455-63.

43. Rondanin R, Simoni D, Romagnoli R, Baruchello R, Marchetti P, Costantini C, Fochi S, Padroni G, Grimaudo S, Pipitone RM, et al. Inhibition of activated STAT5 in Bcr/Abl expressing leukemia cells with new pimozide derivatives. Bioorg Med Chem Lett. 2014;24(18):4568-74.

\section{Publisher's Note}

Springer Nature remains neutral with regard to jurisdictional claims in published maps and institutional affiliations.

Ready to submit your research? Choose BMC and benefit from:

- fast, convenient online submission

- thorough peer review by experienced researchers in your field

- rapid publication on acceptance

- support for research data, including large and complex data types

- gold Open Access which fosters wider collaboration and increased citations

- maximum visibility for your research: over $100 \mathrm{M}$ website views per year

At $\mathrm{BMC}$, research is always in progress.

Learn more biomedcentral.com/submission 